\title{
Cronología del valle del Upano (Alta Amazonía ecuatoriana)
}

Chronologie de la vallée de l'Upano (haute Amazonie équatorienne)

Chronology of the Upano Valley (upper Ecuadorian Amazonia)

\section{Stéphen Rostain}

\section{(2) OpenEdition}

\section{Journals}

Edición electrónica

URL: http://journals.openedition.org/bifea/1825

DOI: $10.4000 /$ bifea.1825

ISSN: 2076-5827

Editor

Institut Français d'Études Andines

Edición impresa

Fecha de publicación: 1 diciembre 2010

Paginación: 667-681

ISSN: 0303-7495

Referencia electrónica

Stéphen Rostain, « Cronología del valle del Upano (Alta Amazonía ecuatoriana) », Bulletin de l'Institut français d'études andines [En línea], 39 (3) | 2010, Publicado el 01 junio 2011, consultado el 07

diciembre 2020. URL : http://journals.openedition.org/bifea/1825; DOI : https://doi.org/10.4000/bifea 1825

\section{(c) (†) $\odot$}

Les contenus du Bulletin de l'Institut français d'études andines sont mis à disposition selon les termes de la licence Creative Commons Attribution - Pas d'Utilisation Commerciale - Pas de Modification 4.0 International. 


\title{
Cronología del valle del Upano (Alta Amazonía ecuatoriana)
}

\author{
Stéphen Rostain*
}

\section{Resumen}

El proyecto arqueológico Sangay-Upano/Río Blanco, realizado entre 1996 y 2003, permitió establecer una secuencia cultural del valle del Alto Upano, desde 700 a. C. hasta nuestros días. Los primeros habitantes del valle pertenecieron a la cultura Sangay. Entre 500 y 200 a. C., las comunidades de la cultura Upano edificaron complejos de montículos a lo largo de los barrancos. Progresivamente, la cerámica Upano, caracterizada por la decoración de bandas rojas entre incisiones, fue reemplazada por la cerámica de estilo Kilamope. Hacia 400-600 d. C., los habitantes huyeron del valle a causa de una fuerte erupción del Sangay. Entre 800 y 1200 d. C., grupos de la cultura Huapula ocuparon algunos de los montículos preexistentes. Son los antepasados de los jívaros actuales que siguen viviendo ahora en la región.

Palabras clave: Amazonía, Upano, Huapula, Jívaro, precolombino, montículo

\section{Chronologie de la vallée de l'Upano (haute Amazonie équatorienne)}

\section{Résumé}

Le projet Sangay-Upano/Río Blanco, mené entre 1996 et 2003, a permis de définir une séquence culturelle de la vallée du haut Upano qui débute vers 700 av. J.-C. et continue jusqu'à nos jours. Les premiers habitants de la vallée appartenaient à la culture Sangay. Entre 500 et 200 av. J.-C., les communautés de culture Upano édifièrent des complexes de monticules le long des ravins. Progressivement, la céramique Upano, caractérisée par le décor de bandes rouges entre incisions, fut remplacée par la poterie de style Kilamope. Vers 400-600 apr. J.-C., les habitants fuirent la

* UMR 8096, ARCHAM, CNRS. Nanterre-Cedex (París, Francia). E-mail: stephen.rostain@mae.uparis10.fr 
vallée à cause d'une forte éruption du Sangay. Entre 800 et 1200 apr. J.-C., des groupes de la culture Huapula occupèrent quelques tertres préexistants. Ce sont les ancêtres des Jivaros actuels qui continuent à vivre dans cette région.

Mots clés: Amazonie, Upano, Huapula, Jivaro, précolombien, monticule

\title{
Chronology of the Upano Valley (upper Ecuadorian Amazonia)
}

\begin{abstract}
The archaeological project Sangay-Upano/Río Blanco, conducted between 1996 and 2003, allowed the definition of a cultural sequence for the upper Upano Valley that begins about 700 BC and continues up to the present. The first inhabitants belonged to the Sangay culture. Between 500 and 200 BC, communities of the Upano culture built mounds complexes along the cliffs. The Upano pottery, characterized by red banded incised decoration, was gradually replaced progress by Kilamope ceramics. About 400-600 AD, inhabitants fled the valley as a result of a strong eruption of Sangay volcano. Between 800 and $1200 \mathrm{AD}$, groups belonging to the Huapula culture settled on some preexisting mounds. These groups are the ancestors of the modern Jivaros, who still live in the region.
\end{abstract}

Key words: Amazonia, Upano, Huapula, Jivaro, pre-Columbian, mound

\section{INTRODUCCIÓN}

Hace dos años, en esta misma revista, fue descrita la última fase de la ocupación precolombina del valle del Upano en la Alta Amazonía ecuatoriana, demostrando que la cultura Huapula era una cultura proto Jívaro y representaba la primera aparición de ese tipo en la cuenca del río Upano. Ello permitió anticipar de algunos siglos la presencia de este grupo en la región (Rostain, 2006). Cabe describir ahora lo sucedido durante los dos milenios que precedieron la llegada de los jívaros y, más específicamente, la secuencia cerámica y cultural del valle del Upano, dividida en cuatro fases.

\section{EL VALLE ARQUEOLÓGICO DEL UPANO}

Encerrado entre dos cordilleras al pie de los Andes, el valle del Upano forma una entidad geográfica en la Alta Amazonía (fig. 1). La cuenca del río Upano es también una entidad cultural que data de la época precolombina, es decir, desde hace casi 3000 años. Varias culturas se han sucedido, desarrollándose en un biotopo específico, ubicado al borde de las tierras bajas amazónicas y las montañas andinas. Esta posición fronteriza es el origen de un poblamiento particular en el que se encuentran rasgos mezclados, amazónicos y andinos.

1 Agradezco a Belém Muriel la revisión del castellano. 
El volcán Sangay domina el valle del Upano al norte y su actividad volcánica permanente influencia considerablemente la vida de los habitantes. Si bien sus cenizas fertilizan el suelo, permitiendo hasta tres cosechas de maíz por año, sus erupciones pueden destruir todo alrededor. El río Upano dibuja una línea recta norte-sur, paralela a los Andes y a la cordillera del Cutucú que la separa de la Baja Amazonía. La característica de este río, ancho y torrentoso, es la ribera formada por abruptos barrancos de 50 a $100 \mathrm{~m}$ de altura. La falla sísmica del Subandino ha formado varias terrazas a orillas del río donde están implantados los sitios arqueológicos.

De 1996 a 2001, se llevó a cabo una cooperación científica franco ecuatoriana, bajo el auspicio del Instituto Francés de Estudios Andinos, cuyo interés era el estudio de los sitios con montículos artificiales de tierra prehistóricos, ubicados en las terrazas altas que bordeaban el río Upano. La particularidad de estos sitios reside en su localización en las faldas de los Andes, en una región intermedia y fronteriza entre montaña y selva. Así, los establecimientos prehistóricos del valle del Upano son una mezcla de rasgos andinos y amazónicos. Las influencias de la sierra se hallan presentes en la edificación de montículos artificiales de tierra, la difusión de cerámicas en el seno de una extensa red comercial, el

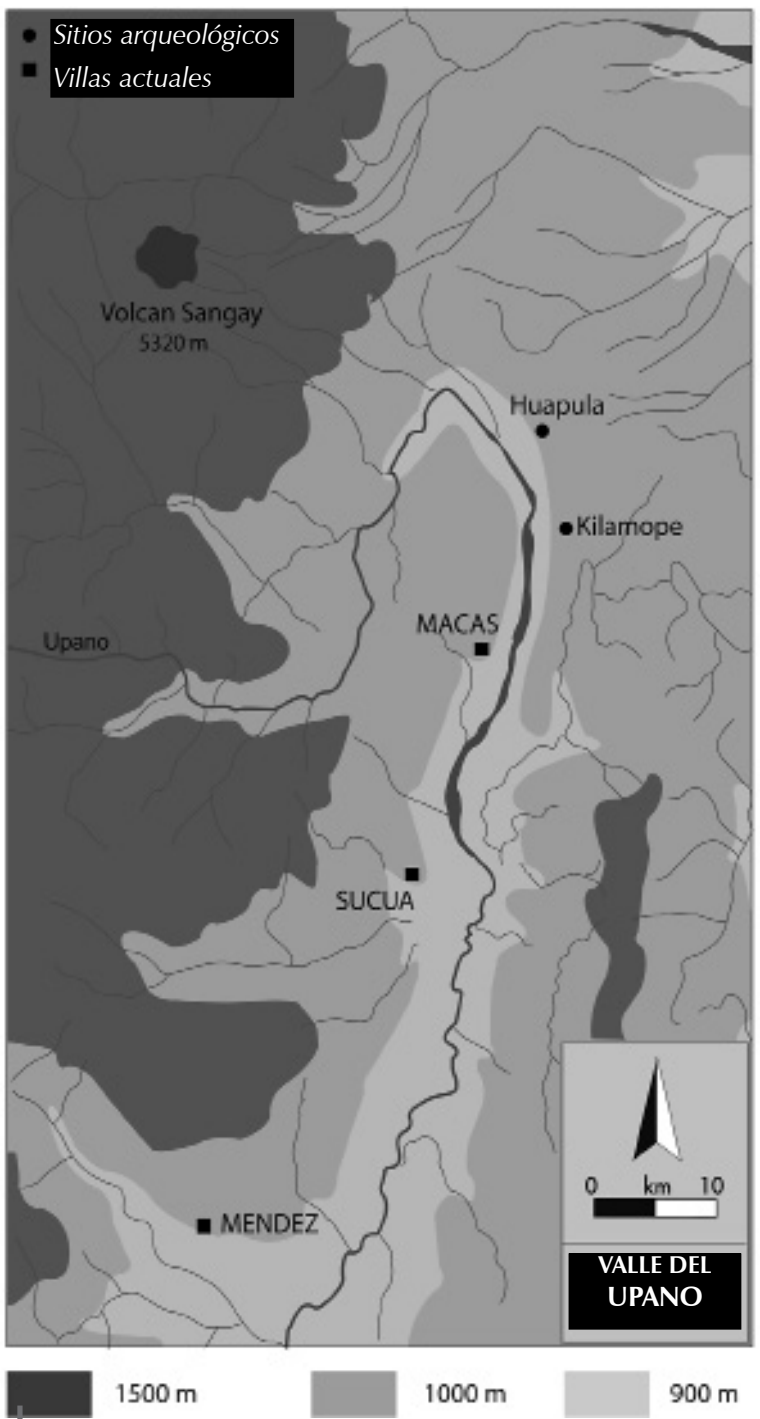

Figura 1 - El valle del Upano con la ubicación de los dos sitios excavados por decapado durante el proyecto Dibujo: S. Rostain uso de metates de piedra pulida y de grandes ollas para preparar la cerveza de maíz. Por otra parte, se pueden apreciar numerosas características de las culturas selváticas amazónicas, entre ellas la ubicación de los sitios a orillas de un río, la técnica de fabricación y el decorado de la cerámica. El mapa arqueológico regional, la organización espacial interna de los sitios y de los montículos y el estudio de las antiguas culturas del Alto Upano forman parte de las investigaciones realizadas en el marco del programa Sangay-Upano/Río Blanco. Hasta ahora habían sido excavados únicamente los sitios con montículos. 
Hace falta precisar que, en las terrazas del Upano, se han encontrado también numerosos sitios sin evidencias de acondicionamiento del terreno, que contienen vestigios de los mismos estilos descubiertos en los montículos. La excavación de estos sitios simples ha permitido establecer paralelos entre los dos tipos de establecimientos, comparar sus cronologías respectivas y explicar sus interrelaciones. Parte del trabajo de campo se concentró en un grupo de montículos del sitio de Huapula —Complejo n. ${ }^{\circ} \mathrm{XI}$ en el inventario de Porras (1987)—. Una excavación por decapado en áreas se hizo en toda la cima de un montículo y en una plaza baja. Diversos interrogantes orientaron el trabajo: ¿Cómo fueron construidos los montículos y bajo qué plan de distribución? ¿Cuál era su función? ¿Son éstos obra de una o varias comunidades? ¿En qué época fueron ocupados? ¿Cómo estaba organizado el espacio en la cima de las plataformas?

Con 70 hectáreas, Huapula es el sitio más extenso del Alto Upano y bordea un afluente del río del mismo nombre. Está compuesto por varias agrupaciones de montículos sobre planicies separadas por profundas quebradas. El sitio principal se compone de unos treinta complejos de montículos, con un gran complejo central de montículos largos y altos. Estos complejos están distribuidos dentro de una red de largos canales profundos y otros secundarios. Tres lugares se excavaron en el sitio de Huapula: i) La Lomita junto al barranco del río, ii) sobre un montículo con doble plataforma del complejo central; y iii) en el Complejo periférico número XI. La plataforma XI bordea el río Huapula y se extiende en un área de $3500 \mathrm{~m}^{2}$. Está organizado según el modelo espacial característico de los sitios del Upano. Es decir, una plaza central dividida en dos por una plataforma central y rodeada por seis elevaciones periféricas. En este complejo, algunas áreas han sido excavadas a mano: en la cima del montículo central y en una plaza (Rostain, 1999a, b, c). Se han realizado además varios sondeos. La estratigrafía es muy interesante (fig. 2): sobre el nivel estéril, hay una primera ocupación bajo el relleno de construcción coronado por un suelo quemado y una ocupación; después viene un nivel espeso de cenizas volcánicas anterior a un último nivel de ocupación.

Otras excavaciones por decapado y sondeos se realizaron en un nuevo sitio ubicado algunos kilómetros más al sur, constituido por un complejo de cinco montículos Ilamado Kilamope (fig. 2). Las prospecciones demostraron que los alrededores de este complejo fueron ocupados por los antiguos habitantes en un área estimada de 500 m de diámetro. La estratigrafía de Kilamope era comparable a la de Huapula, con varios niveles de ocupación separados por la construcción de los montículos y una capa de cenizas volcánicas del Sangay. A pesar de esta similitud, los datos obtenidos en este sitio completaron los de Huapula con nuevas informaciones. Además de estas dos excavaciones, sondeos y prospecciones fueron realizados en numerosos complejos de montículos y sitios sin estructura de tierra.

El sitio de Huapula fue previamente excavado durante la década de 1970 por Pedro Porras (1987), quien volvió famoso el sitio al interpretar la disposición de los montículos centrales como un jaguar copulando con un hombre. El nuevo mapa del sitio hecho en 1997 demostró la ausencia de esta imagen. Pedro 

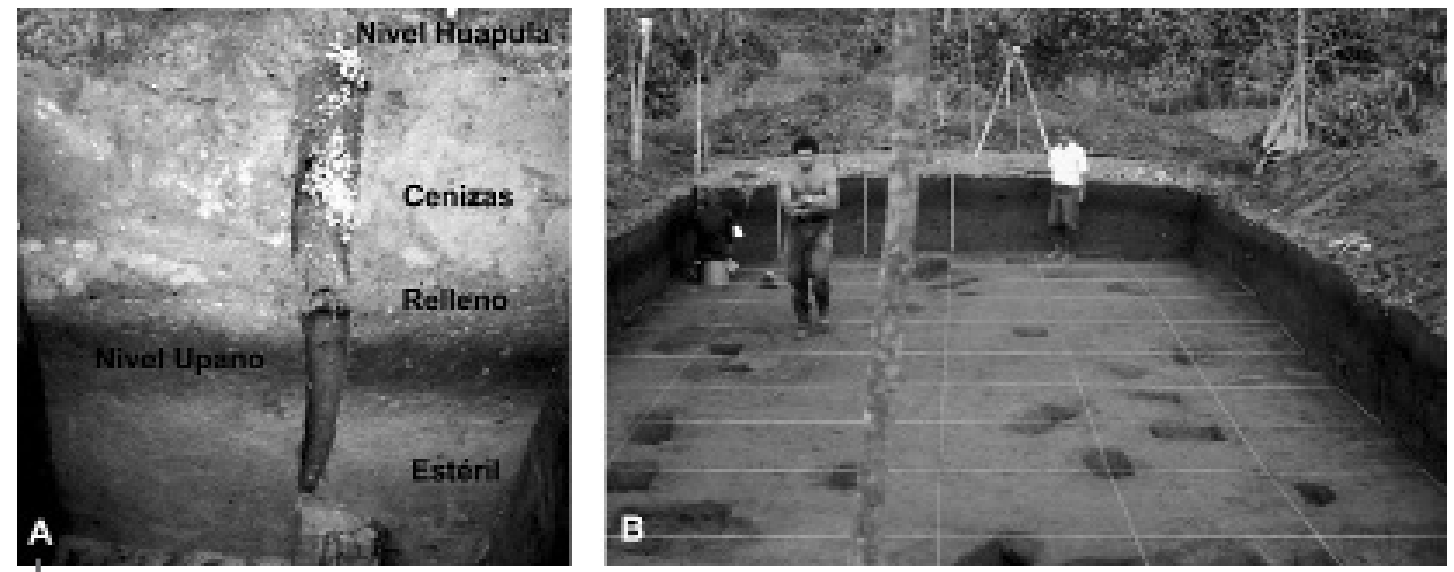

Figura 2A - Estratigrafía de la tola central del Complejo XI del sitio de Huapula. B. Superficie decapada en un montículo periférico del sitio de Kilamope

Fotos: S. Rostain

Porras reconoció en el valle del Upano una sola tradición cultural de 3500 años, llamada Upano, pero su clasificación cerámica estaba basada únicamente en las decoraciones por una parte y las formas, por otra; sin hacer referencia a la estratigrafía y a las fechas de radiocarbono. El gran número de tipos definidos y las superposiciones de algunos de ellos impiden el uso de esta tipología. Una revisión basada en nuevas excavaciones era necesaria, la misma que permitió definir cuatro fases culturales.

Una gran colección de tiestos fue recogida durante las excavaciones y las prospecciones, proporcionando una muestra completa de la secuencia cerámica del valle del Upano. Se definió una nueva tipología en base a la pasta, la decoración y la forma de la cerámica, cruzada con la estratigrafía y las fechas de radiocarbono. Además, se estudiaron varias colecciones museográficas, ayudando a la reconstitución de las formas cerámicas. Gracias a la colaboración del museo del Banco Central de Guayaquil, los recipientes recogidos durante las excavaciones fueron restaurados por Julio Burgos (fig. 3).

La estratigrafía de los montículos y de los alrededores, las fechas de radiocarbono y la tipología cerámica permitieron la definición de una secuencia del valle del Alto Upano que empezó alrededor del 700 a. C., prolongándose hasta la fecha.

\section{LA CULTURA SANGAY}

Pocos vestigios de la primera ocupación fueron recogidos durante las excavaciones en el valle del Upano. Alrededor de 700 a. C., algunas comunidades de la cultura Sangay se instalaron a orillas del Upano. Los primeros en llegar ocuparon directamente el espacio sin realizar terraplenes. La cerámica Sangay era burda y frágil, mal cocida, de color gris-blanco, con un desgrasante de pequeña grava lisa y 
paredes finas. La decoración era sencilla y lo común era la aplicación de cordones ondulados o botones aplicados e incisiones simples (fig. 4). Las formas reconocidas, platos y ollas redondas de boca cerrada, presentaban a veces abultamientos de la pared. En un recipiente, estos lóbulos incisos verticalmente representaban rostros de animales que se asemejan a una calabaza (fig. 4).

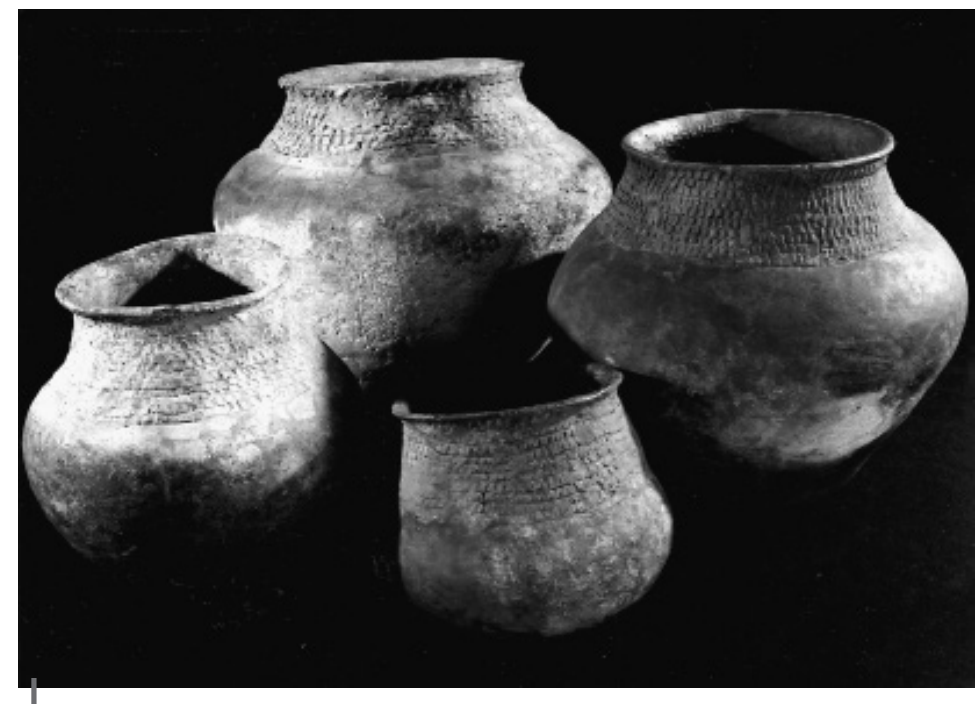

Figura 3 - Ollas con cuello corrugado Huapula descubiertas en la cima de la tola central del Complejo XI de Huapula

Foto: R. Jones

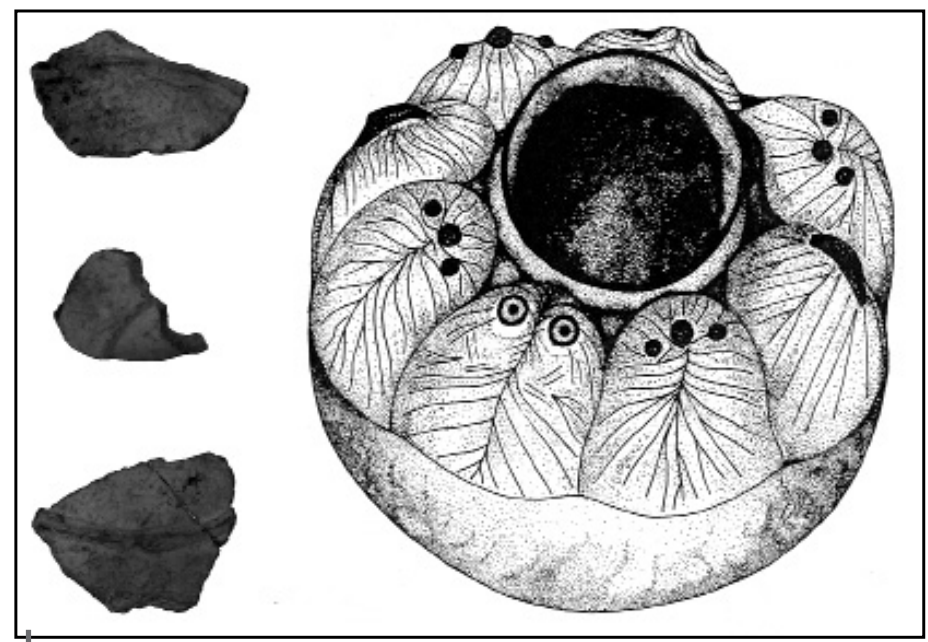

Figura 4 - Cerámica Sangay con los cordones ondulados aplicados del sitio de Huapula y pequeña olla con caras incisas (Porras, 1987: 208) Fotos: S. Rostain 


\section{LA CULTURA UPANO}

El inicio de la cultura Upano se sitúa entre 500 y 200 a. C. Los upano han construido a lo largo del valle montículos de tierra, plazas bajas, canales y caminos cavados, distribuidos en complejos con un modelo espacial preciso (fig. 5). En varios casos, han aprovechado el relieve natural del lugar para edificar la estructura. A causa del suelo lodoso y resbaladizo, han quemado la cima de los montículos de arcilla obteniendo así un suelo compacto. El modelo de distribución es una plaza central cerrada por montículos. Sirve de base para variaciones, incluyendo una plataforma central o más montículos periféricos. Ciertos caminos cavados que conducen a un riachuelo están generalmente asociados a los complejos. Las excavaciones que he realizado en dos sitios indican que la primera función de algunos montículos era habitacional; pero otros, como los estrechos de la periferia, tenían otro uso dado que era imposible edificar una casa en su cima. Es probable que los complejos hayan sido al mismo tiempo un lugar doméstico y ceremonial.

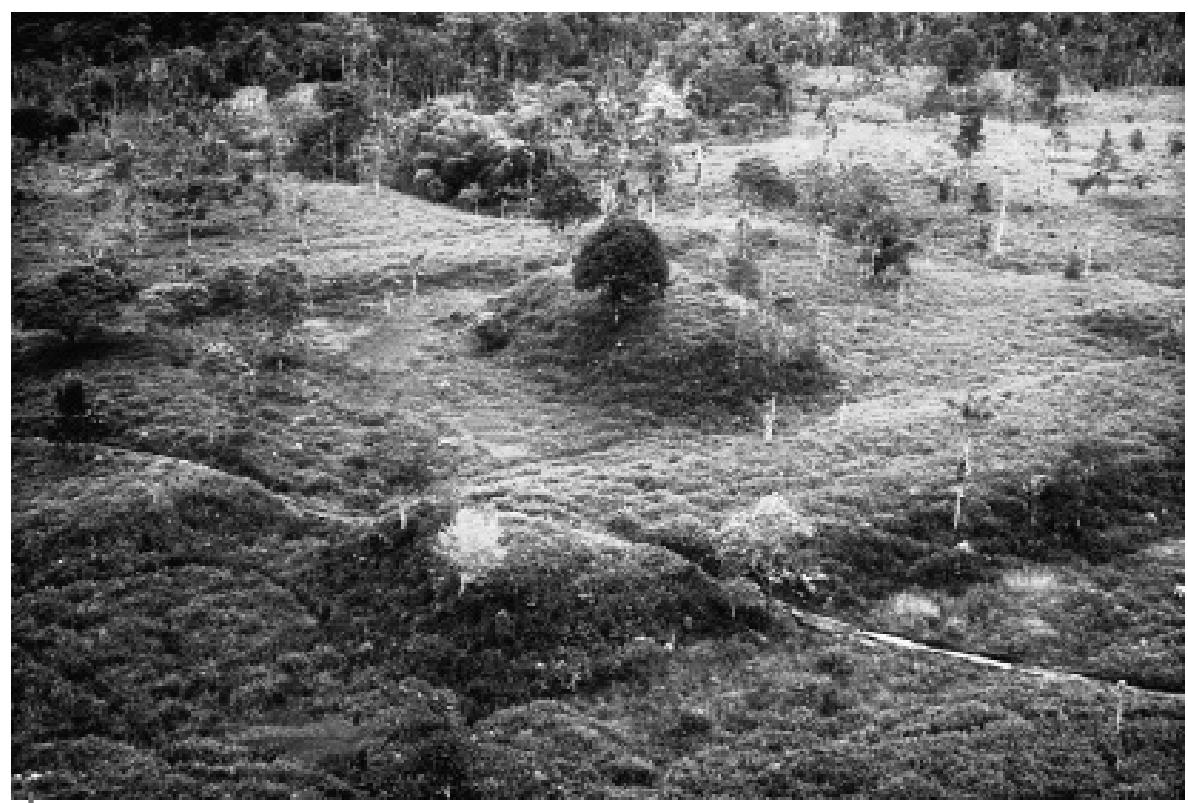

Figura 5 - Complejo de montículos de Eden-1

Foto: S. Rostain

La cerámica Upano es homogénea, bien cocida, de color beige claro o café oscuro y con desgrasante de arena fina. Las características de la pasta la diferencian claramente de la cerámica Sangay. Pedro Porras ha definido una multitud de tipos decorativos que impiden una visión clara del material. Además, estos tipos no eran exclusivos ya que los mismos tiestos aparecían en diferentes tipos. 
El tipo de las bandas rojas entre incisiones es la decoración característica de la cerámica Upano (fig. 6). Se la identifica por su pintura roja entre líneas rectas o curvas incisas, finas o gruesas. A veces, se encuentra pintura negra, café y blanca entre las incisiones. En algunos casos, la pintura roja no está sobre la pared del recipiente sino dentro de la incisión. Los motivos geométricos son muy diversos incluyendo bandas rectas, triángulos, rectángulos, espirales, etc. A pesar de una gran variedad de motivos, el tipo de bandas rojas entre incisiones se reconoce inmediatamente. Las bandas anchas alternan con las finas. Los mismos motivos pueden estar solamente pintados de rojo sin las incisiones al lado. Incisiones, punteados o botones aplicados pueden acompañar la decoración. Las formas de recipiente también son muy diversas: platos, cuencos, ollas, cazuelas... Algunos presentan formas muy complicadas resaltando la habilidad de los alfareros upano. La mayoría de las formas corresponden a recipientes para comer y beber, mientras que otras han servido para conservar líquidos o alimentos. Una forma recurrente es la olla con cuerpo globular abierto, cuello cerrado y borde horizontal salido. El borde está típicamente decorado por una serie de triángulos rojos. La forma cerámica upano más popular es el plato, que conoce numerosas variaciones (fig. 6). Puede ser redondeado o con fondo plano y paredes verticales rectas o sinuosas. El diámetro varía de 15 a $30 \mathrm{~cm}$ y la altura de 5 a $15 \mathrm{~cm}$. La decoración puede ser una simple incisión horizontal, con motivos rojos o bandas rojas entre ellas. Estos platos, probablemente usados para comer o beber, fueron producidos en enormes cantidades. A pesar de que aparecen generalmente en la literatura como platos con fondo plano y sin base, tienen en realidad varios tipos: anular, pie troncocónico o tres botones. Como en otros recipientes upano, y sobre todo del tipo de bandas rojas entre incisiones, los platos tienen el interior bruñido en negro brillante. El borde interior presenta generalmente de una a tres incisiones anchas paralelas y a veces, el fondo tiene incisiones anchas poco profundas con motivos simples, eventualmente con botones.

La incisión es el segundo tipo de decoración más común de la cerámica Upano (fig. 7). Son incisiones finas o anchas, punteados, achurado. Las incisiones son en su mayoría líneas rectas simples con motivos paralelos o triangulares. El tercer tipo upano es la pintura negativa negra sobre rojo (fig. 7). Son series de bandas negras paralelas que forman motivos triangulares o en rombos. Las formas son cuencos globulares o vasos. Parece que este tipo aparece en la temporada tardía de la ocupación Upano.

La decoración Upano está esencialmente hecha por motivos geométricos no figurativos, así que es difícil analizar la iconografía. Hay que anotar la ausencia de adornos en esta cerámica. Excepcionalmente, hay motivos figurativos como una rana aplicada sobre una pared o, a la izquierda una rana y una serpiente excisas y pintadas de rojo (fig. 8). Este tema es interesante porque, en la iconografía amazónica, estos dos animales están asociados a la mujer. Además, la representación de una serpiente mirando (o amenazando) a una rana se encuentra en otras regiones amazónicas. Es común en las culturas de la tradición polícroma de la desembocadura del Amazonas, en el borde de recipientes funerarios de la cultura Aristé de la costa del Amapá en Brasil y en Guyana Francesa (Rostain, 1994: fig. 101). 
Cronología del valle del Upano (Alta Amazonía ecuatoriana)

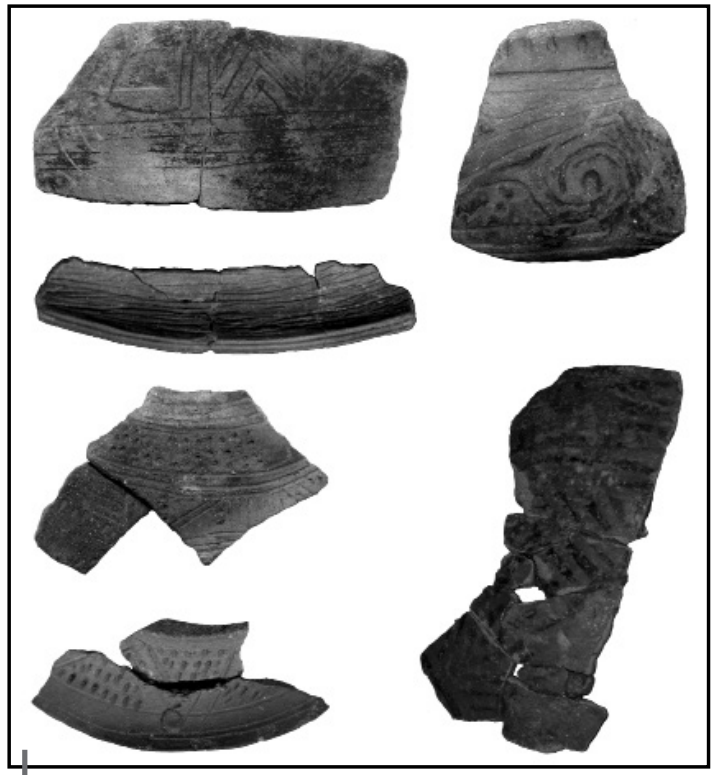

Figura 7 - Cerámica Upano incisa y en negativo del sitio de Huapula

Fotos S. Rostain

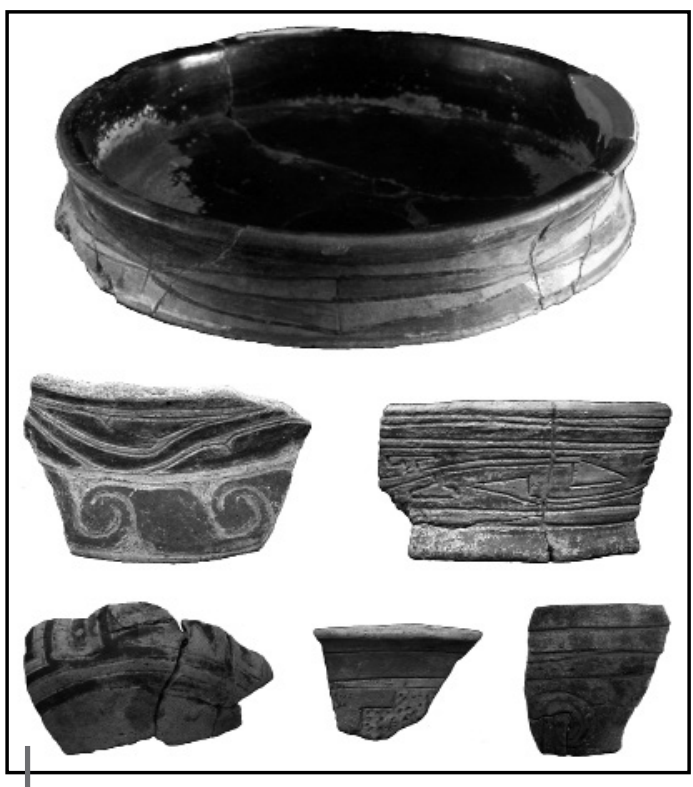

Figura 6 - Cerámica Upano de bandas rojas entre incisiones de los sitios de Huapula y de Kilamope Arriba, plato característico con el interior bruñido. El último tiesto presenta incisiones pintadas de rojo.

Fotos: S. Rostain

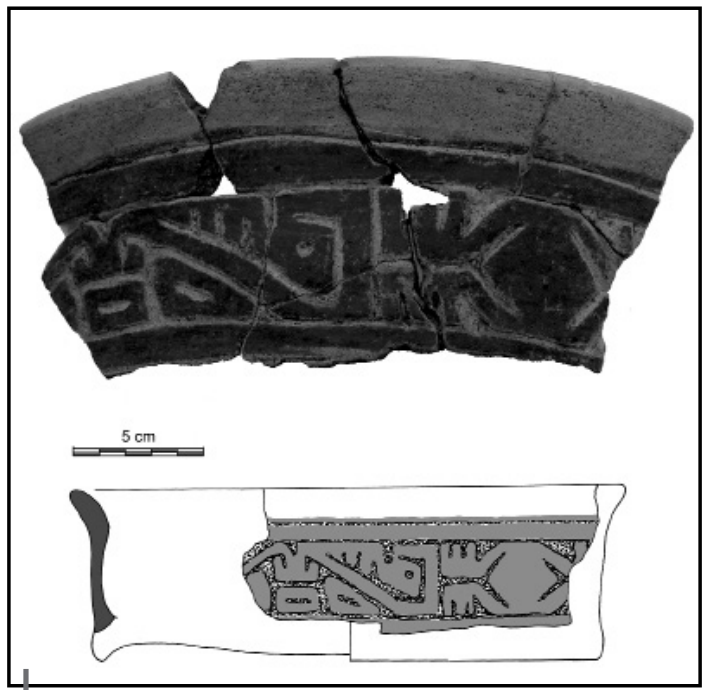

Figura 8 - Cerámica Upano excisa y pintada de rojo, con el motivo de la rana y de la serpiente del sitio de Kilamope

Foto y dibujo: S. Rostain 
Desde el inicio de la ocupación Upano, se dieron estrechos intercambios con la sierra inmediata al oeste del valle. En el sitio de Pirincay, se hallaron numerosos tiestos de bandas rojas entre incisiones en un nivel fechado entre 400 y 100 a. C. Los análisis mineralógicos comprobaron que esta cerámica fue fabricada con arcilla del valle del Upano (Bruhns et al., 1994). Tipos exógenos fueron descubiertos puntualmente en el sitio de Huapula donde se notó la presencia de cerámicas de la cultura serrana Panzaleo. Así, un tiesto del tipo «cáscara de huevo» se halló en la tola central del Complejo XI de Huapula y algunos tiestos Panzaleo aparecieron también en las excavaciones de «La Lomita» del mismo sitio (Pazmiño Tamayo, 2008). Esto confirmó la existencia de intercambios con culturas vecinas. El tiempo y energía necesarios para la construcción de los complejos, la jerarquización de los sitios y los intercambios a larga distancia son la prueba de una sociedad organizada en jefatura.

El área de extensión de la cerámica Upano de bandas rojas entre incisiones está bien delimitada al norte por el río Pastaza, al este por la cordillera del Cutucú y al oeste por los Andes. Hacia el sur, conoce una distribución más amplia porque aparece a lo largo del curso del Upano (Rostoker, 2005) y del Santiago en Perú, donde se encuentran varios sitios Upano. Su presencia es reconocida hasta el río Ucayali, donde el tipo de bandas rojas entre incisiones aparece en la cerámica Cumancaya (Lathrap, 1970; Roe, 1973). La fecha tardía del 800 d. C. de esta cultura sugiere que los upano llegan a la región después de su desaparición en el valle del Upano.

\section{LA CULTURA KILAMOPE}

Después de la ocupación Upano, se destaca la cultura Kilamope que se instala en los mismos sitios. Su cerámica presenta la misma pasta que la de Upano, al igual que una gran variedad de formas. Existen muchos casos de mezcla de motivos y formas Upano y Kilamope en un solo recipiente, lo que conduce a pensar que la cultura Kilamope goza de la influencia y la integración de otra cultura externa a la cultura Upano, que podría originarse en la cultura Pastaza (Saulieu, 2006; Saulieu \& Rampón Zardo, 2006).

La decoración más común de la cerámica Kilamope es la incisión y excisión (fig. 8), pero tiene muchas técnicas asociadas y una multitud de motivos geométricos más elaborados que los upano. La decoración más típica es la impresión cordelada (fig. 9): una línea ancha y profunda hecha con la impresión de una cuerda o con el vaivén de un punzón que imita el dibujo de una cuerda. Los motivos son líneas cortas y largas, paralelas. La decoración cordelada está generalmente asociada a otras técnicas decorativas: incisión, punteado y pintura. Por ejemplo, el cuenco de la figura 9 presenta una asociación característica de cordelado e incisiones, mientras que el interior está decorado con pintura negativa café con fondo beige, muy diferente del negativo Upano. Las bandas anchas forman motivos paralelos rectos y curvos. Los platos Upano desaparecen en la cerámica Kilamope para 
ser reemplazados por vasos con base plana y paredes rectas verticales. Como en el estilo Upano, hay muchas formas muy elaboradas y originales. La cerámica Kilamope no se ha documentado fuera del valle del Upano.

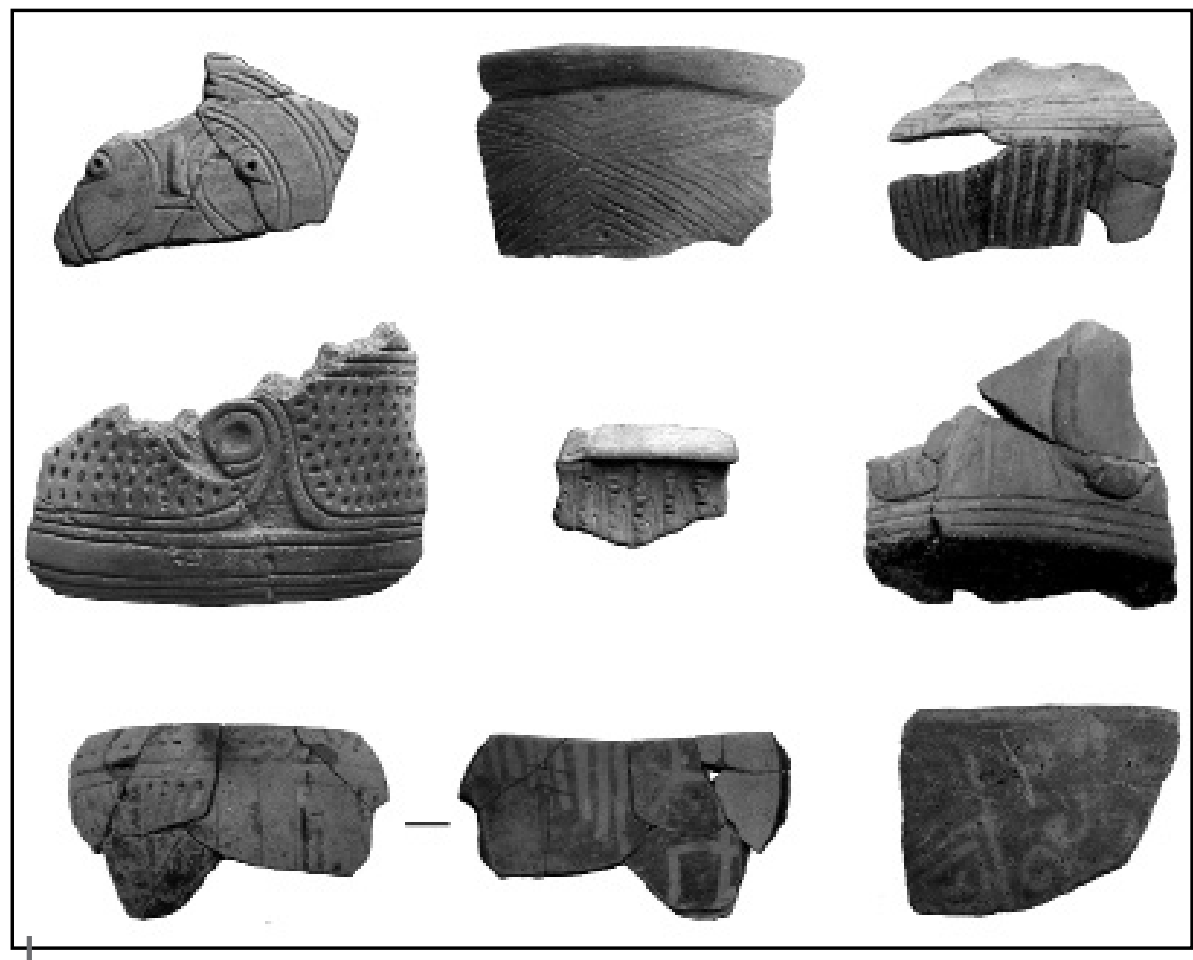

Figura 9 - Cerámica Kilamope del sitio de Kilamope con varios decorados: incisiones, cordelado, puntuado, aplicados, negativo

Abajo, a la izquierda, un cuenco con decoración exterior cordelada, incisa y puntuada y decoración interior pintada en negativo.

Fotos: S. Rostain

Hacia 400-600 d. C., una erupción del Sangay depositó una gruesa capa de cenizas en el valle del Upano propiciando la huida de los habitantes. Fue una erupción de cenizas y no una nube ardiente, que destruyó los asentamientos. Podemos recalcar que la capa de cenizas ha sido muy espesa en el sitio de Huapula, con 30 $\mathrm{cm}$ de espesor, y va disminuyendo progresivamente hacia el sur hasta desaparecer en Sucúa. Después de esta catástrofe, los upano no volvieron al valle. Ciertos indicios sugieren que llegaron hasta el río Ucayali en el Perú. 


\section{LA CULTURA HUAPULA}

Entre 800 y 1200 d. C., llegaron al valle grupos de la cultura Huapula, que se asentaron sobre algunos montículos preexistentes. Esta ocupación se encontró en todo el valle del Upano pero fue mucho menos densa que la ocupación Upano. La excavación por decapado de la cima del montículo de la tola central en el sitio de Huapula reveló un suelo habitacional muy bien conservado. El análisis espacial de los rasgos y vestigios permitió identificar la práctica de diversas actividades y su distribución en el área de la casa. El estudio etnoarqueológico de esta habitación mostró las grandes similitudes existentes con el modo de hábitat actual de los grupos jívaros (Rostain, 2006). La cocina estaba localizada en el medio de la habitación, con un grupo principal de fogones que se extendían en $4 \mathrm{~m}^{2}$. El área ocupada por estos últimos, las piedras molares y las ollas de cerámica representaban más o menos $15 \mathrm{~m}^{2}$, es decir, un sexto de la superficie total. Los restos de las cuatro ollas corrugadas grandes, dos cuencos simples y algunas cerámicas se juntaban exactamente al norte de los fogones centrales (Rostain, 2000).

La cerámica precolombina más reciente es de cultura Huapula. La pasta se caracteriza por un desgrasante de arena gruesa con granos de cuarzo y un color gris o café oscuros. Las formas conocidas son cuencos globulares y grandes y pequeñas ollas globulares con cuello recto vertical (fig. 10). Los dos tipos de decoración son el corrugado y la pintura roja y blanca. Excepcionalmente, hay pintura negra y roja. Los motivos son bandas finas paralelas y puntos sobre pequeños recipientes; y la calidad de su pasta se diferencia de las grandes ollas corrugadas. Las paredes son más finas y de color beige, mientras que el desgrasante arenoso es más fino.

Grandes ollas globulares con un cuello recto y corrugado fueron descubiertas en varios sitios del valle del Upano. El corrugado pudo ser hecho de simples bandas aparentes o con impresión de dedo o de uña. En la tola central de Huapula, habían cinco ollas Huapula completas o casi completas (una estaba ubicada en el extremo sur del montículo y sirvió para tapar una fosa de almacenamiento). El exterior de estas ollas estaba cubierto por una espesa capa de hollín. Un residuo de alimento, pegado en la pared interna de un tiesto de olla, presentaba microestrías propias de la superficie de los granos de maíz. Esto demostró que el maíz era uno de los alimentos que este recipiente contenía. Si bien el maíz se cocinaba de múltiples formas, al parecer los huapula lo utilizaban sobre todo para la elaboración de la chicha, una suave cerveza espesa. Inestables debido a su base redonda, las ollas debían estar aseguradas para no voltearse. $\mathrm{Al}$ igual que en las casas amerindias, estaban parcialmente enterradas en una de las cuatro fosas encontradas en la casa. En la cima de una fosa, se hallaba solamente la parte superior de una gran olla cortada horizontalmente debajo de la espalda del recipiente. Se supone que esta mitad de cerámica cerraba un pozo, probablemente usado para conservar productos alimenticios. Como lo demostró Jean Guffroy (2006), la decoración corrugada tiene una extensa difusión, incluyendo Venezuela, Colombia, el Ecuador, el Perú, Bolivia y hasta el sur del Brasil. 


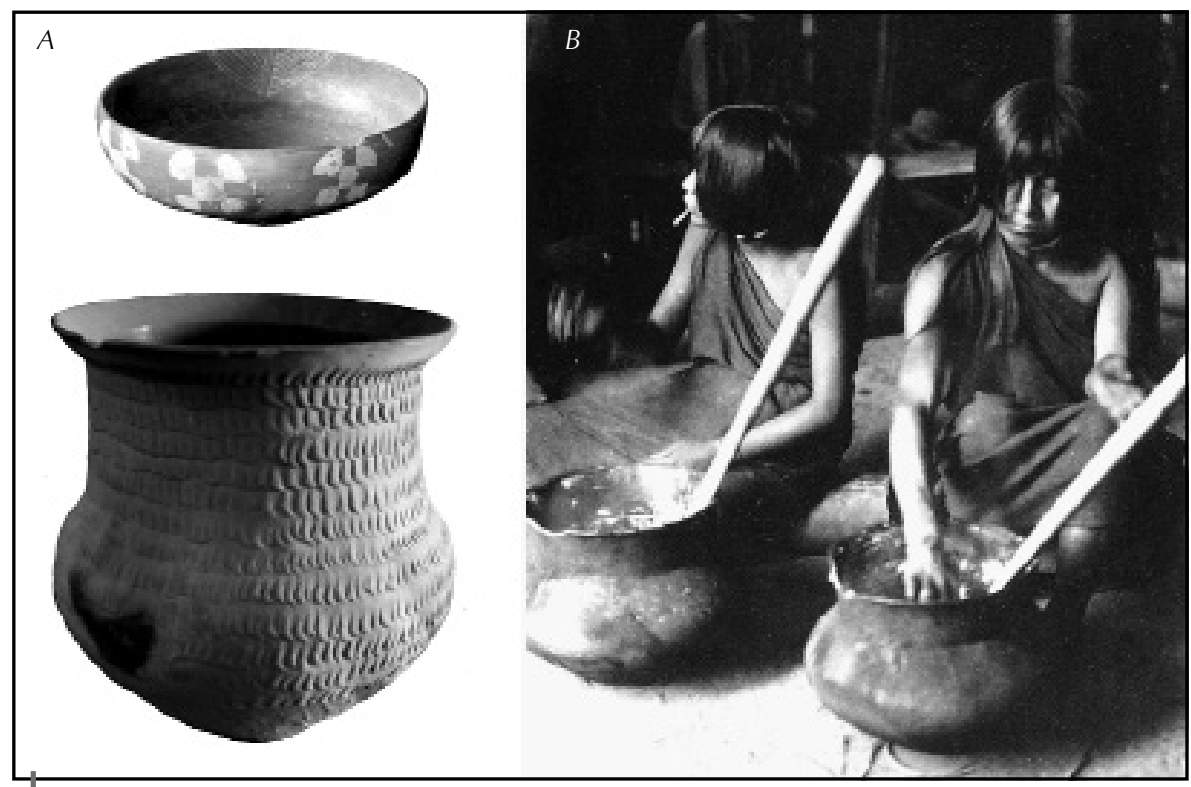

Figura 10A - Cerámicas pintada en blanco y corrugada jívaras

Fotos: S. Rostain

10B. Shuar haciendo chicha de maíz en 1935 (foto anónima)

En el caso presente, la filiación de la cerámica Huapula y Jívaro no tuvo duda: las ollas corrugadas y los recipientes pintados con bandas blancas y rojas fueron los antepasados de la cerámica jívara actual.

\section{LA CULTURA JÍVARO}

La presencia del grupo shuar de cultura Jívaro está comprobada en el valle del Upano desde los primeros cronistas (Bianchi ed., 1982; Descola, 1986; 1993; Harner, 1995; Renard Casevitz et al., 1986; Taylor \& Landazúri, 1994): han llegado a esta región más de 500 años antes de la Conquista española. Así que este grupo y su cerámica están presentes desde hace un milenio, aproximadamente, en muchos lugares del valle.

La cerámica jívara se caracteriza por dos categorías principales (fig. 10): primero, existen ollas para preparar la chicha, cocinar la comida o las hojas de tabaco; segundo, hay cuencos grandes para comer o beber la chicha. La decoración es sencilla, con bandas corrugadas o motivos simples de bandas anchas pintadas.

Los jívaros consumen gran cantidad de chicha, cerveza muy espesa que se sirve a menudo de almuerzo. Se muelen los granos en metates y la harina que se obtiene es mezclada con agua, siendo el agente de fermentación la harina masticada. La chicha se prepara en grandes ollas de cerámica y se ofrece en cuencos del 
mismo material o de calabaza. Se elaboran grandes cantidades para las fiestas comunitarias donde se ingiere un número impresionante de cuencos.

\section{CONCLUSIÓN}

Las excavaciones arqueológicas realizadas con anterioridad en la Amazonía consistían en pequeños sondeos estratigráficos. Durante el proyecto SangayUpano/Río Blanco, adopté una nueva técnica de excavación decapando grandes superficies. Ello me permitió obtener una visión global del suelo arqueológico e interpretar más precisamente los rasgos y vestigios dejados por los antiguos habitantes del lugar.

Si con cuatro culturas sucesivas - Sangay, Upano, Kilamope y Huapula-, la historia precolombina del valle del Upano parece más compleja de lo que se pensaba, la persistencia de comunidades durante siglos en esta misma región demuestra una estabilidad notable.

\section{Referencias citadas}

BIANCHI, C. (ed.), 1982 - Artesanías y técnicas shuar, 477 pp.; Quito: Ediciones Mundo Shuar. BRUHNS, K. O., BURTON, J. H. \& ROSTOKER, A., 1994 - La cerámica «incisa en franjas rojas»: evidencia de intercambio entre la Sierra y el Oriente en el Formativo tardío del Ecuador. In: Tecnología y Organización de la Producción Cerámica Prehispánica en los Andes (Shimada, ed.): 53-66; Lima: Pontifica Universidad Católica del Perú.

DESCOLA, P., 1986 - La nature domestique. Symbolisme et praxis dans l'écologie des Achuar, 450 pp.; París: Fondation Singer-Polignac, Éditions de la Maison des Sciences de I'Homme.

DESCOLA, P., 1993 - Les lances du crépuscule. Relations Jivaros, Haute Amazonie, 506 pp.; París: Plon. Colección Terre Humaine.

GUFFROY, S., 2006 - El Horizonte corrugado: correlaciones estilísticas y culturales. Bulletin de l'Institut Français d'Études Andines, 35 (3): 347-360; Lima.

HARNER, M. J., 1995 - Les Jivaros: Hommes des cascades sacrées, 198 pp.; París: Petite Bibliothèque Payot 264.

LATHRAP, D. W., 1970 - The Upper Amazon, 256 pp.; Londres: Thames and Hudson.

PAZMIÑO TAMAYO, E. M., 2008 - Análisis cerámico del sitio La Lomita, Morona Santiago, Ecuador, 110 pp.; Quito: PUCE. Disertación de Licencia no publicada.

PORRAS, P., 1987 - Investigaciones arqueológicas a las faldas del Sangay, 432 pp.; Quito: Centro de Investigaciones Arqueológicas, PUCE.

RENARD-CASEVITZ, F.-M., SAIGNES, T. \& TAYLOR-DESCOLA, A.-C., 1986 - L'Inca, l'Espagnol et les Sauvages : rapports entre les sociétés amazoniennes et andines du XV au XVII siècle, 411 pp.; París: Éditions Recherche sur les Civilisations. Synthèse 21 . 
ROE, P. G., 1973 - Cumancaya: Archaeological Excavations and Ethnographic Analogy in the Peruvian Montaña; Urbana: Department of Anthropology, University of Illinois. Unpublished Ph.D. dissertation.

ROSTAIN, S., 1994 - L'occupation amérindienne ancienne du littoral de Guyane; París: Éditions de I'ORSTOM. Colección Travaux et Documents Micro-fichés 129.

ROSTAIN, S., 1999a - Excavación en área de un montículo de Huapula, proyecto Sangay-Upano. In: Memorias del Primer Congreso Ecuatoriano de Antropología, vol. 3: 227-256; Quito: Museo Jacinto Jijón y Caamaño, PUCE, MARKA.

ROSTAIN, S., 1999b - Secuencia arqueológica en montículos del valle del Upano en la Amazonía ecuatoriana. Bulletin de l'Institut Français d'Etudes Andines, 28 (1): 1-37; Lima.

ROSTAIN, S., 1999c - Occupations humaines et fonction domestique de monticules préhistoriques en haute Amazonie équatorienne. Bulletin de la Société Suisse des Américanistes, 63: 71-95; Neuchâtel.

ROSTAIN, S., 2000 - Analyse d'un sol d'habitat précolombien en Amazonie équatorienne. In: Peuplements anciens et actuels des forêts tropicales (Froment \& Guffroy, eds.): 142-146; Orléans: Laboratoire ERME, IRD.

ROSTAIN, S., 2006 - Etnoarqueología de la casa Huapula y Jívaro. Bulletin de I'Institut Français d'Etudes Andines, 35(3): 337-346; Lima.

ROSTOKER, A., 2005 - Dimensions of prehistoric human occupation in the southern ecuadorian Oriente, 613 pp.; Nueva York: University of New York. Unpublished PhD.

SAULIEU, G. de, 2006 - Revisión del material cerámico de la colección Pastaza (Amazonía ecuatoriana). Journal de la Société des Américanistes, 92 (1-2): 279-302; París.

SAULIEU, G. de \& RAMPÓN ZARDO, L., 2006 - Colección arqueológica de MoronaSantiago del Museo Amazónico de la Universidad Politécnica Salesiana, 115 pp.; Quito: Abya-Yala.

TAYLOR, A.-C. \& LANDÁZURI, C., 1994 - Conquista de la Región Jívaro (1550-1650), 471 pp.; Quito: Marka, IFEA, Abya-Yala. Fuente para la Historia Andina 4, Travaux de l'IFEA 83. 